\title{
Kastration von Hund und Katze - eine Betrachtung aus ganzheitlicher Sicht
}

Gisela Bolbecher und Brigitta Smit-Fornahl für den ethischen Zirkel AKSENT

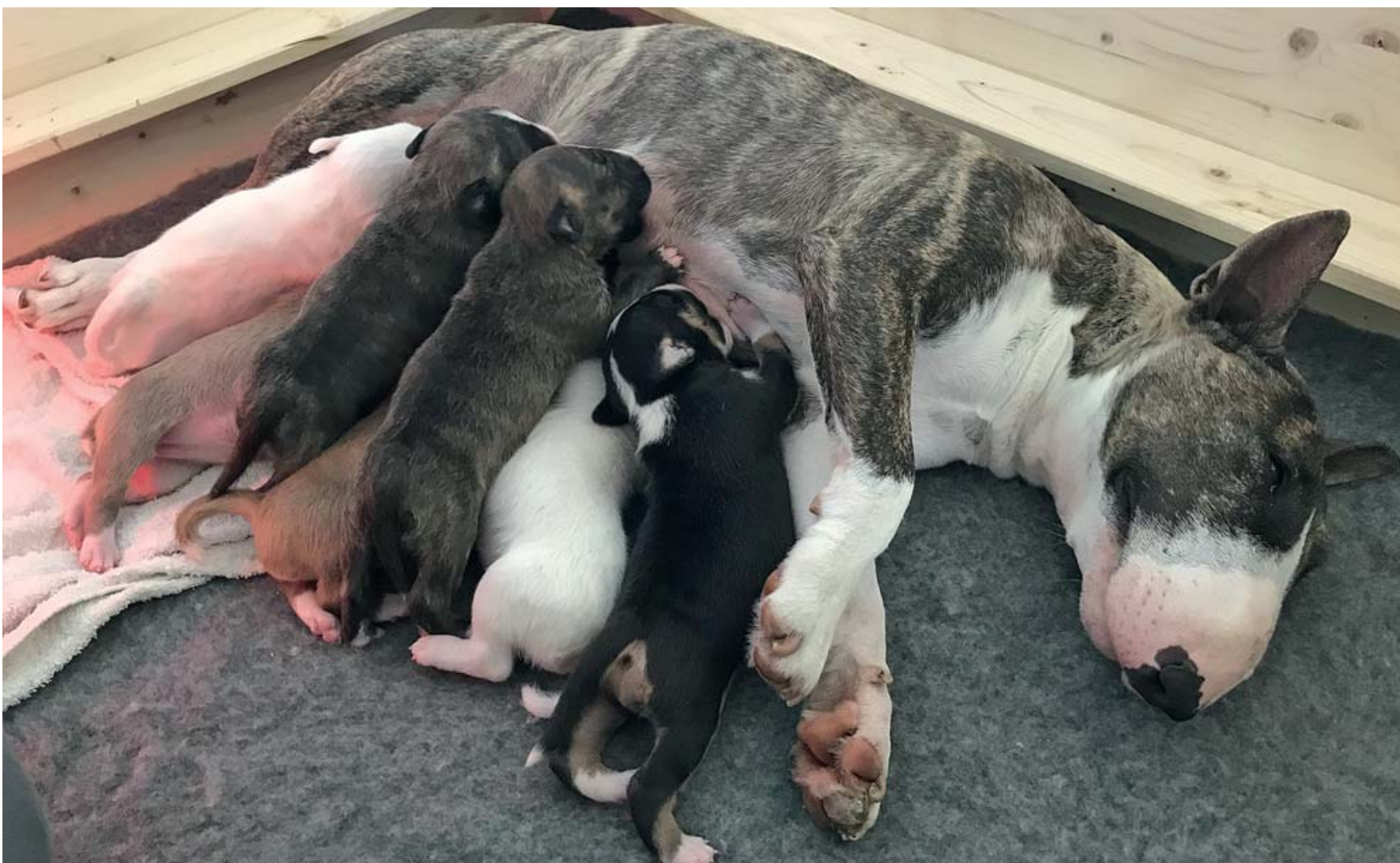

Hündin mit Welpen. @ Kathrin Meinlschmidt

Kastrationen gehören zu den häufigsten chirurgischen Eingriffen in der Tiermedizin. Aus ganzheitlicher Sicht stellt dieser Eingriff eine erhebliche Beeinträchtigung des jeweiligen Tieres dar. Die Ansichten über die Indikation sind auch in der Tierärzteschaft uneinheitlich. Welche Erkrankungen werden verhindert? Welche Nebenwirkungen sind zu erwarten? Wie sollen wir aus ganzheitlicher Sicht beraten? Das Interview mit Prof. Dr. Wehrend bietet dazu einige Einschätzungen und Diskussionsansätze.

\section{Kastration? Ja, nein, vielleicht?}

Als ganzheitlich denkende und arbeitende Tierärztinnen betrachten wir dieses Thema unter verschiedenen Gesichtspunkten.

Altbekannt sind Studien aus den 1980er und 1990er Jahren mit folgenden Ergebnissen:

- Die möglichst frühe Kastration von Hündinnen kann die Entstehung von Mammatumoren oder Erkrankung des Uterus unterbinden.
- Die Kastration macht einen entspannten Umgang mit Rüden möglich, verhindert Rangordnungsprobleme und Prostataerkrankungen.

Auch in den Köpfen der Hundehalter sitzt die Angst vor solchen Erkrankungen tief und wurde lange Zeit durch sich wiederholende Veröffentlichungen geschürt.

Als Tierärzte stehen wir im Spannungsfeld verschiedener Interessen: zwischen unserem Wissen als Tiermediziner, 
als berufener Tierschützer, der die Interessen der Tiere vertritt, und den Wünschen und Nöten unserer Kunden.

Wie so oft sitzen wir zwischen den Stühlen. Wem sind wir verpflichtet? Welche Faktoren beeinflussen unsere Entscheidung? Welche rechtlichen Rahmenbedingungen spielen eine Rolle?

\section{KASTRATION? JA, NEIN, VIELLEICHT?}

Hundehalter wünschen die Kastration ihres Tieres, weil...

- ... das Zusammenleben einfacher ist.

- ... unerwünschte Trächtigkeiten vermieden werden.

- ...Unannehmlichkeiten bei Läufigkeiten nicht auftreten.

- ...Verhaltensprobleme während der Scheinträchtigkeit vermieden werden.

- ....Aggressionsprobleme bei Rüden sich vermindern.

- ... sie Angst vor dem Auftreten von möglichen Erkrankungen (Pyometra, Mammatumoren, Lactatio falsa) haben.

Der Wunsch, Tiere kastrieren zu lassen, ist in den letzten 20 Jahren erheblich gestiegen. Die Kastration von Katzen und Katern verhindert eine unkontrollierbare Vermehrung und das damit verbundene Leid. Dies hat aus Tierschutzsicht eine über das Individualrecht des einzelnen Tieres hinausgehende Berechtigung, ein sogenannter vernünftiger Grund, wie ihn das Gesetz fordert.

Bei Hunden sieht das anders aus. Unkontrollierte Vermehrung ist in Deutschland weniger verbreitet, die Tiere sind meist in enger Obhut des Menschen. Und doch scheint es, als wenn dieser Eingriff eine „Selbstverständlichkeit" geworden wäre und es beinahe ketzerisch anmutet, wenn ein Halter oder Tierarzt das hinterfragt oder ablehnt. Dabei hat sich das Wissen um die Folgen einer Kastration in den letzten Jahren stark erweitert (s. Kasten 2 und Interview mit Prof. Dr. Wehrend). Folgen der Kastration sind, wie schon lange bekannt, Inkontinenz, Adipositas und Hypothyreose. Hinzu kommt ein höheres Risiko für schwerwiegende Erkrankungen (Neoplasien, Diabetes mellitus usw.). Und darüber hinaus haben neue Studien ergeben, dass bei intakten Geschlechtsorganen keineswegs mehr Neoplasien dieser Organe auftreten.

Das bedeutet, dass wir jeden Einzelfall viel detaillierter und individueller betrachten sollten. In unseren Praxen ist es üblich, für diese Kastrationsgespräche einen entsprechenden Zeitrahmen zu bieten, um die Besitzer umfassend aufzuklären. Dabei geht es auch um die Verantwortung gegenüber den uns anvertrauten Mitgeschöpfen, die in drei richtungsgebenden Papieren niedergelegt ist:
Das Tierschutzgesetz bezeichnet in $\S 1$ das Tier als unser Mitgeschöpf, dessen Leben und Wohlbefinden geschützt werden muss. Wohlbefinden setzt auch Kenntnis dessen voraus und meint nicht die subjektive Meinung eines Tierhalters. Niemand darf ohne einen vernünftigen Grund einem Tier Schmerzen, Leiden oder Schäden zufügen. Bei einer Kastration ohne strenge medizinische Indikation wäre dies der Fall.

Der $§ 6$ regelt u.a. das Entfernen von Organen, welches grundsätzlich verboten ist. Die Einschränkung dieses Verbotes bezüglich der unkontrollierten Fortpflanzung oder der Unfruchtbarmachung zur weiteren Haltung oder Nutzung wird klar von der tierärztlichen Meinung abhängig gemacht.

Der Ethikcodex der Bundestierärztekammer und dessen Umsetzungsanleitung geben noch deutlichere Leitlinien vor. Auch hier wird neben dem Schutz der Gesundheit auch das Wohlbefinden der Tiere als Ziel formuliert. Der Tierarzt vertritt die Interessen der Tiere gegenüber der Gesellschaft, Politik, Wirtschaft und Wissenschaft und soll vorrangig bei Zielkonflikten die Bedürfnisse der Tiere berücksichtigen. Er setzt sein Wissen dafür ein, Tiere vor Schmerzen, Schäden, Leiden und Angstzuständen (siehe Kasten 2 Folgen der Kastration) zu bewahren, Krankheiten zu vermeiden und orientiert sein tierärztliches Handeln am Ziel der Wiederherstellung, des Erhalts oder der Verbesserung der individuellen Lebensqualität. Daneben wird auch die Aufklärung der Tierhalter über die Anschaffung und Haltung eines Tieres durch den Tierarzt hervorgehoben, über die physischen, psychischen und sozialen Bedürfnisse der einzelnen Tierarten zu informieren und damit Leiden durch Unkenntnis vorzubeugen.

Der Codex Veterinarius der TVT richtet sich nach dem Leitsatz „in dubio pro animale“. In seiner Präambel betont der Codex die „ethische Verantwortung“ aller Tierärzte für das empfindungsfähige und leidensfähige Tier und fordert Schutz und Fürsorge nicht nur aufgrund seines Nutzwertes ein. Auch verpflichtet er die Tierärzte zur stetigen Aktualisierung des Wissensstandes, um diese Bedürfnisse erfüllen zu können. Der Gleichheitsgrundsatz bei Bedürfnissen und Interessen von Mensch und Tier ermahnt, die Interessen der Menschen nicht grundsätzlich höher zu bewerten.

Diese Rahmenbedingungen sind bei jeder Diskussion, ob Kastration sinnvoll ist oder nicht, zu berücksichtigen und unterstreichen den Stellenwert von Tieren und ihr Dasein als Mitgeschöpfe. In der Regulationsmedizin stellt diese Haltung die Grundlage für unser Handeln und unsere Therapien dar. 


\section{Interview mit Prof. Dr. Axel Wehrend zum Thema Kastration bei Hunden und Katzen}

Was hat dazu geführt, dass Sie sich kritisch mit der Thematik Kastration auseinandergesetzt haben?

Prof. Dr. Wehrend: Ich würde dies nicht als kritische Haltung bezeichnen, sondern in meiner Funktion als Fachtierarzt und Leitung der klinischen Reproduktionsmedizin ist eine offene Haltung und die ganzheitliche Sichtweise bei dem Thema Kastration notwendig. Was bewirkt der Wegfall der Sexualsteroide? Dies kann nicht nur in Bezug auf die Sexualorgane beurteilt werden, da dieser Einfluss auf den gesamten Organismus haben. Das Tier in seiner Gesamtheit sollte im Fokus stehen.

Warum hat sich diese Sichtweise verändert?

Prof. Dr. Wehrend: Früher standen die Auswirkungen auf die Sexualorgane im Vordergrund, im Rahmen des Erkenntnisgewinns ist das Wissen über die Wirkungen der Sexualsteroide, z.B. auf das Immunsystem, Verhalten und auf die Zunahme von neoplastischen Erkrankungen, sehr stark angestiegen. Außerdem haben Hundebesitzer in früheren Jahren nicht nach den Folgen der Kastration gefragt, Tierärzte haben sich mit dieser Fragestellung wenig beschäftigt. Heute hat der Bedarf an Aufklärung bei den Hundebesitzern stark zugenommen und es ist notwendig, differenziertere Antworten zu geben.

In Ihrem Artikel in der „Tierärztlichen Praxis Kleintiere 4/2017 haben Sie viele Erkrankungen beschrieben, die Folge der Kastration von Hündinnen sein können. Gibt es schon Erkenntnisse über den Einfluss der Kastration beim Rüden?

Prof. Dr. Wehrend: Die wahrscheinlichen Auswirkungen der Kastration bei Rüden auf andere Erkrankungen werden rechnerisch ermittelt, so ist für einen kastrierten Rüden die Wahrscheinlichkeit, einen Diabetes mellitus zu entwickeln, um das 1,9-fache erhöht. Allerdings sind andere Risikofaktoren noch höher, entscheidend ist dafür auch der Kastrationszeitpunkt. Je früher Rüden kastriert werden, desto mehr steigt das Risiko. Dazu gibt es viele neue Daten und es bleibt abzuwarten, ob diese in weiteren Studien bestätigt werden können.

Das Risiko für eine neoplastische Erkrankung (Blutzellen, Osteosarkome, Übergangszellkarzinome) steigen bei kastrierten Rüden an. Das Risiko für ein Prostatakarzinom steigt hingegen durch eine Kastration an, wenn auch diese Erkrankung eher selten auftritt. Die Beratung in der Praxis im Hinblick auf das erhöhte Risiko ist sehr schwierig. Bei einem Boxer, der an sich schon sehr tumorphil ist, steigt durch die Kastration das Risiko, z. B. einen Mastzelltumor oder eine Inkontinenz zu entwickeln.
Bei einer therapeutischen Kastration besteht eine absolute Indikation, wie z. B. ein Hodentumor oder Ovartumor. Anders verhält es sich bei einem relativen Risiko. Hier gilt es abzuwägen, ob die Kastration indiziert ist oder ein nebenwirkungsarmes Medikament zur Verfügung steht. Ein Beispiel soll dies erläutern: Ein Rhodesien Ridgeback Rüde soll nach Wunsch des Züchters der Zucht erhalten bleiben und nimmt deswegen an etlichen Ausstellungen erfolgreich teil. Im Alter von 4 Jahren entwickelt er eine Prostatahyperplasie, der Besitzer lässt auf Anraten des Tierarztes den Hund sofort kastrieren. Ein halbes Jahr später meldet sich der Züchter, da eine passende Hündin gefunden ist, die er decken soll. Der Konflikt ist vorprogrammiert - wie ist diese Situation zu klären? War die Kastration die einzige Lösung? Kastration als Therapie kann auch eine schwierige Lösung sein.

Bei Katzen ist die Kastration It. TSG erlaubt. Gibt es bei diesen Tieren Erkenntnisse über die Auswirkungen der Kastration auf die Lebenserwartung und Folgeerkrankungen? Wann ist hier der optimale Zeitpunkt für die Kastration?

Prof. Dr. Wehrend: Die Kastration bei Katzen hat deutlich weniger Nebenwirkungen als bei Hunden. Je früher die Kastration stattfindet - zwischen 4. und 7. Monat -, desto größer ist die Chance, dass die kastrierte Kätzin keine Mammatumore entwickelt. Kätzinnen werden aus zwei Gründen kastriert: Es geht um die Vermeidung von unkontrollierter Fortpflanzung, v.a. wenn die Kätzin in einem Mehrkatzenhaushalt mit unkastrierten Katern lebt. Der Zeitpunkt ist dann abhängig von der Geschlechtsreife und sollte vor Eintritt der Geschlechtsreife durchgeführt werden. Bei Einzelhaltung von Katzen kann die Kastration solange hinausgezögert werden, bis sich die typischen sexuell motivierten Verhaltensweisen einstellen. Nach der Kastration kommt es zu einer Umstellung im Stoffwechsel und zur Gewichtszunahme. Viele Katzen neigen dazu und die Besitzer sind angehalten, die Ernährung anzupassen.

Die Überlebenszeit von kastrierten Kätzinnen (Freigang) ist im Durchschnitt um 1 Jahr höher und die von kastrierten Katern um 4 Jahre. Es sind die innerartlichen Aggressionen und Territorialkämpfe, verbunden mit Traumata und Stress, die bei kastrierten Tieren deutlich zurückgehen. Infolgedessen sinkt auch die Wahrscheinlichkeit, sich an einer der typischen Katzenviruserkrankungen zu infizieren und zu sterben.

Ist die Kastration von Hunden mit dem Tierschutzgesetz und dem Codex veterinarius vereinbar, wenn Besitzer wegen ihres sozialen Umfeldes (Zweithund, Tierpension, Bequemlichkeit, Angst) dies verlangen?

Prof. Dr. Wehrend: Es gibt im TSG §6 (1) 3 mögliche Gründe, wann Tiere kastriert werden dürfen. Medizinische (tierärztliche Indikation), jagdliche Nutzung, Kontrolle der Fortpflanzung und wenn keine tierärztlichen Bedenken der Unfruchtbarmachung zur weiteren Hal- 
tung entgegenstehen. Je umfangreicher unser tierärztliches Wissen ist, desto mehr spielen diese Bedenken eine Rolle. Bei Katzen gibt es deutlich weniger Vorbehalte hinsichtlich einer Kastration als bei Hunden. Wenn Menschen Tiere in ihre persönliche Obhut nehmen, ist stets die Frage zu stellen, ob ich es dem Tier gestatte sich ganz auszuleben. Hier gibt es zwei Positionen. Aus Sicht der Tierrechtler gestehe ich dem Tier gleiche Grundrechte zu wie Menschen. Die körperliche Unversehrtheit ist zu erhalten, jede Kastration ist abzulehnen. Aus Sicht der Tierschützer leben Hund und Katze mit Menschen zusammen und jeder macht dabei Kompromisse. Wenn ich der Kastration einer Hündin zustimme, nehme ich in Kauf, dass die kastrierte Hündin eine Harninkontinenz entwickelt. Ein Zustand, bei dem ich davon ausgehe, dass sich eine Hündin, die auf Sauberkeit erzogen wurde, nicht wohl fühlt. Die Behandlung mit Sympathomimetika führt häufig zu Herzrasen. Sie therapiert das Symptom Harninkontinenz, verursacht aber bei dem kastrierten Tier einige Nebenwirkungen. Die Frage ist, darf ich diese Nebenwirkungen einem Tier zumuten - ein ethisches Problem.

An unserer Klinik werden viele Kastrationsberatungen durchgeführt und 40\% unserer Kunden entscheiden sich gegen eine Kastration. Ideal wäre eine Beratung vor dem Kauf eines Hundes, damit die Kunden sich im Klaren sind, was auf sie zukommt. Wenn ich mir z. B. einen Rüden anschaffe, sollte es mir von vorn herein klar sein, dass Rüden markieren. Wenn ich das nicht will, kann ich alternativ ein kastriertes Tier aus dem Tierheim wählen.

Wenn in unserer Klinik Tiere mit Harninkontinenz vorgestellt werden, empfehlen wir den Besitzern immer, wegen der Nebenwirkungen der Therapie mit Sympathomimetika mit ganzheitlichen Therapien anzufangen und verweisen sie an die Haustierärzte.

Können Sie sich vorstellen, in diesem Fall an Tierärzte aus der GGTM zu verweisen, da viele Haustierärzte aufgrund ihrer Ausbildung über keine ausreichenden Kenntnisse über ganzheitliche Therapieangebote verfügen?

Prof. Dr. Wehrend: Ja. Wir haben schon einige Vorträge mit der GGTM zusammen gestaltet, was von den Studenten gut angenommen wurde.

Gibt es eine Dokumentationspflicht über die Aufklärungsgespräche mit den Besitzern?

Prof. Dr. Wehrend: Es ist notwendig, bei dem Beratungsgespräch auf die Nebenwirkungen der Kastration hinzuweisen. Wer dies nicht tut und die Besitzer im Unklaren über die möglichen Folgen lässt, hat seinen Job nicht gemacht. Dies sollte auch immer schriftlich fixiert werden.

Frage: Wie häufig sind Ihrer Erfahrung nach Hypothyreosen nach Kastration? In der Praxis beobachten wir dies sehr oft, besonders bei Labrador und Retriever.
Prof. Dr. Wehrend: Der Einfluss der Kastration auf Schilddrüsenerkrankungen ist aus Sicht der Studien nicht sehr hoch. Aus Sicht des Praktikers scheint die Hypothyreose häufiger bei kastrierten Hündinnen aufzutreten, allerdings auch bei Zuchthündinnen. Labrador und Retriever machen einen großen Anteil der Patienten aus und scheinen eine Prädisposition zu haben. Hundebesitzer lassen ihre Hündin wegen der Angst vor Gebärmutter- und Ovartumoren kastrieren.

\section{MÖGLICHE FOLGEN EINER KASTRATION}

Positiv:

- Erkrankungen der Ovarien, Eileiter oder Uterus sind nicht mehr möglich (Inzidenz bei nicht kastrierten Hündinnen abhängig von Rasse und Kastrationsalter 2-20\%), aber geringer protektiver Effekt auf die Entstehung von Mammatumoren bei Kastration vor 2. Läufigkeit.

- Verlängerte Lebenserwartung

Negativ:

- Uterusstumpfpyometra

- Vulvapyodermie bei Adipositas

- Deutliche Zunahme von Tumorentwicklung im Thorax, Atemwegen, Magen-Darm-Trakt, Weichteilgewebe, endokrine Drüsen

- Mastzelltumore mit Rasseprädisposition (Rhodesian Ridgeback, Boxer, Labrador, Retriever, Mops, Viszla, DSH)

- Lymphome mit Rasseprädisposition Schweizer SH, Viszla, Rottweiler, Labrador, DSH

- Hämangiosarkome (DSH, Retriever, Labrador, wenig pigmentierte und kurzhaarige Hunde)

- Übergangszellkarzinome der Blase

- Osteosarkome bei großen Hunderassen

- Harninkontinenz mit Inzidenz von 5-20\% in Abhängigkeit von Gewicht, Rasse, Adipositas, Rutenkopierung, Kastrationszeitpunkt

- Negative Beeinflussung des Immunsystems mit erhöhtem Risiko für atopische Dermatitis, autoimmunhämolytische Anämie, Hypothyreoidismus, immunvermittelte Thrombozytopenie, chronisch entzündliche Darmerkrankungen und Lupus erythematosus

- Einfluss auf den Stoffwechsel mit Adipositas, chronische Pankreatitis mit möglichen Diabetes mellitus und Hypothyreose

- Bei Frühkastration eunuchoider Hochwuchs

- Erhöhtes Risiko von Kreuzbandrissen und Hüftgelenksdysplasie sowie Patellaluxation

- Saisonale Flankenalopezie und Fellveränderungen, Haarwechselprobleme

- Verhaltensauffälligkeiten wie Angst vor Gewitter und Geräuschen, evtl. Aggression 


\section{BERATUNGSGESPRÄCH ZUM THEMA KASTRATION IN DER PRAXIS}

Als Tierärzte haben wir die Möglichkeit, schon vor dem Kauf eines Hundes oder bei Erstvorstellung im Welpenalter über die Vorzüge und Herausforderungen zu informieren, die ein Rüde oder eine Hündin mit sich bringen. Hier können die ersten Grundsteine für eine umfassende Beratung des Besitzers gelegt werden, auch um Fehlinformationen aus verschiedenen Richtungen (Internet, Hundeschulen, Nachbarschaft) keinen Raum zu bieten. Der Hundehalter möchte in den Entscheidungsprozess eingebunden und mit seinen Ängsten (z. B. vor Tumoren) ernst genommen werden.

Die Fragen, die wir stellen, beinhalten viele Informationen über das soziale Umfeld, weitere Tiere im Haushalt, Kenntnisse in Hundehaltung und Erziehung, Erfahrungen mit früheren Tieren und deren Erkrankungen...

All das führt zu dem Punkt, an dem der Besitzer vor uns steht, und wir die entsprechenden Informationen für die Entscheidung Rüde/Hündin oder Kastration geben sollen:

- Warum haben Sie sich für einen Rüden/für eine Hündin entschieden?

- Welche Erfahrungen haben Sie mit Hunden?

- Leben weitere Hunde, und wenn ja welchen Geschlechtes, im Haushalt?

- Gab es mit früheren Tieren im Zusammenhang mit dem Geschlecht Probleme? Zum Beispiel Scheinträchtigkeiten, unerwünschte Trächtigkeit, Tumorerkrankungen des Gesäuges oder bei Rüden Rangordnungsaggressionen, Hypersexualität, Prostataerkrankung?

- Gab es starke emotionale Beeinträchtigungen, z. B. depressionsartige Stimmungslagen, Futterverweigerung, Weglaufen oder Jaulen und Winseln?

- Welche Probleme befürchten Sie im Zusammenhang mit Pubertät, Geschlechtsreife, Läufigkeit und Scheinträchtigkeit?

- Was sind Ihre Gründe für eine Kastration?

- Haben Sie Informationen über mögliche Folgen eines solchen Eingriffs?

- Kennen Sie mögliche Alternativen zur Kastration?

Hier ist es unsere Aufgabe, abzuwägen zwischen den möglichen Vorteilen für Tier und Mensch im alltäglichen Zusammenleben, und der Wahrscheinlichkeit von unerwünschten Folgen der Kastration, in Abhängigkeit der Rasse, des Alters zum Kastrationszeitpunkt, des körperlichen Zustandes des Hundes und Gewichtes.

Als ganzheitlich arbeitende Tierärzte fügen wir alle Informationen aus diesem Hund-Mensch-Feld zusammen und können vielfältige Lösungsangebote zur Prävention und Therapie womöglich auftretender Probleme anbieten:

- Regulation der belastenden Symptome rund um die Läufigkeit, Scheinträchtigkeiten auf körperlicher und psychischer Ebene mit Phytotherapie, Organotherapie und Homöopathie

- Beruhigung von Verhaltensauffälligkeiten rund um männliches, überschießendes Verhalten

- Regulation von Prostataerkrankungen

- Therapeutische Angebote für Verhaltensauffälligkeiten im hormonellen Zusammenhang

- Empfehlung von geeigneten und informierten Hundetrainern und Hundeschulen

Was glauben Sie, hätte Mutter Natur sich dabei gedacht, wenn es zuträfe, dass bei unkastrierten Hündinnen vermehrt Tumore und Erkrankungen rund um die Sexualorgane auftreten?

Prof. Dr. Wehrend: Wir müssen zwischen dem Wildhund und dem Haustierhund unterscheiden. Der Wildhund ist monoöstrisch, wird einmal im Jahr läufig, wird gedeckt und ist trächtig. Die Lebenserwartung ist deutlich niedriger. Haustierhunde werden in der Regel zweimal läufig pro Jahr, werden häufig nicht gedeckt, haben mehr Läufigkeiten als der Wildhund. Mit der Anzahl der Zyklen steigt die Wahrscheinlichkeit für Entzündungen und neoplastische Erkrankungen. Durch die Domestikation unterscheiden sich Haustiere auch im Bereich der Sexualorgane von den Wildtieren, da eine erhöhte Fruchtbarkeit mit großen Würfen und häufigem Zyklieren ein angestrebtes Zuchtziel war und ist. Um diesen neuen Erkenntnissen über die möglichen Folgen einer Kastration Rechnung zu tragen, sind Hundehalter und der Tierarzt gehalten, Hundeschulen auszuwählen und zu empfehlen, in denen eine Kastration der Tiere nicht vorgeschrieben ist. Gleiches gilt für Hundepensionen. Wenn diese die Kastration als Bedingung für die Aufnahme der Hunde stellen, ist dies aus Sicht der Tiergesundheit abzulehnen. Die Tierhalter sollten sich ggfls. nach Alternativangeboten umsehen.

Die Kastrationspflicht, die manche Tierschutzvereine für Hunde fordern, ist aus rechtlicher Sicht nicht haltbar.

Herr Professor Wehrend, wir bedanken uns für dieses informative und ausführliche Gespräch. 


\section{ZUSAMMENFASSUNG}

Die in den letzten Jahren scheinbar gängige Praxis, Haustiere grundsätzlich zu kastrieren, sollte unter Einbeziehung neuer Studien über die Folgen eines solchen Eingriffes und unter Berücksichtigung der ethischen und gesetzlichen Rahmenbedingungen, neu überdacht werden. Der kompetenten und einfühlsamen Beratung der Tierhalter durch uns Tierärzte kommt damit eine große Bedeutung zu.

\section{SUMMARY}

Neutering of dogs and cats: Considerations from a holistic perspective

The apparently common practice of neutering pets in recent years should be reconsidered, taking into account new studies on the consequences of such an intervention as well as the ethical and legal general conditions. The consultation of the pet owners by qualified and empathic veterinarians is therefore of great importance.

\section{Korrespondenzadresse}

\section{Dr. Gisela Bolbecher}

Atzelsberger Str 10

91094 Bräuningshof

bolbecher@die-tierischen.de

Brigitta Smit-Fornahl

Bahnhofstr. 16

58579 Schalksmühle

happy-animals@gmx.de

\section{Bibliografie}

DOI https://doi.org/10.1055/a-0750-2124

Zeitschrift für Ganzheitliche Tiermedizin 2018; 32: 132-138

(c) Sonntag Verlag in Georg Thieme Verlag KG Stuttgart .

New York ISSN 0939-7868 